DYMAT 2009 (2009) 909-915

(C) EDP Sciences, 2009

DOI: $10.1051 /$ dymat/2009127

\title{
Evaluation of dynamic compressive properties of PLA polymer blends using split Hopkinson pressure bar
}

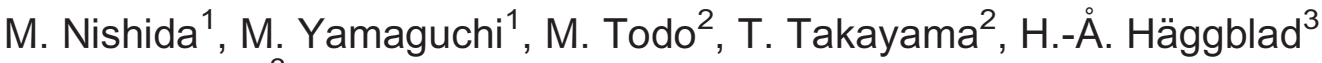 \\ and $\mathrm{P}$. Jonsén ${ }^{3}$ \\ 1 Nagoya Institute of Technology, Gokiso-cho, Showa-ku, Nagoya, Japan \\ 2 Kyushu University, 6-1, Kasuga-koen, Kasuga, Fukuoka, Japan \\ ${ }^{3}$ Luleå University of Technology, 97187 Luleå, Sweden
}

\begin{abstract}
Poly(lactic acid) (PLA) has been used as a biomaterial for bone fixation devices in oral and orthopedic surgery because of good biocompatibility and bioabsorbability. Because Poly $(\varepsilon-$ Caprolactone) (PCL) is a ductile, bioabsorbable and biodegradable polymer, many types of PLA/ PCL polymer blends have been developed to improve its material strength and impact resistance. The stress-strain curves of PLA and PLA/PCL polymer blends were measured using a split Hopkinson pressure bar (Kolsky bar) method and a universal testing machine. The effect of PCL content on Young's modulus and yield stress was examined. The values of constants in CowperSymonds equation with respect to yield stress were determined for PLA and PLA/PCL polymer blend specimens. PLA/PCL specimens were observed using a scanning electron microscope.
\end{abstract}

\section{INTRODUCTION}

Poly(lactic acid) (PLA) shows good biocompatibility and is decomposed and absorbed within a living organism. PLA is widely used for bone fixation materials which do not require second surgery for removal in the field of oral and orthopedic surgery. Because PLA is brittle, its mechanical properties have been improved by polymer blends or natural fiber reinforcing to be used extensively as bone fixation materials and structural materials. Since Poly( $\varepsilon$-Caprolactone) (PCL) is a ductile, bioabsorbable and biodegradable polymer, many types of PLA/PCL polymer blends have been developed to improve its material strength and impact resistance [1-4]. Although good results have been reported, in most cases, the impact resistances of PLA/PCL polymer blends are based only on the experimental results of Izod impact strength test/Charpy impact strength test and Dynatup impact test/dart impact test [5]. The basic mechanical properties of these polymer blends with respect to impact resistances are still unknown. Also, these mechanical properties have not yet been fully elucidated at high strain rates.

In the present study, stress-strain curves of PLA and PLA/PCL polymer blend specimens were measured at room temperature using a split Hopkinson pressure bar (Kolsky bar) method at high strain rates and using a universal testing machine at low strain rates. PLA/PCL specimens were observed using a scanning electron microscope. The effect of PCL content on stress-strain curves at high strain rates was discussed.

\section{EXPERIMENTAL METHODS}

\subsection{Specimens}

We used PLA and blends of PLA/PCL fabricated using PLA from Shimadzu Co., Ltd. (Lacty ${ }^{\circledR}$ \#5000) and PCL from Daicel Chemistry Industries Co. (Celgreen P-H7). The average molecular 
weight of the PLA pellets is $M_{\mathrm{w}}=2.0 \times 10^{5} \mathrm{~g} / \mathrm{mol}$, the glass transition temperature $T_{\mathrm{g}}=60^{\circ} \mathrm{C}$ and the melting point $T_{\mathrm{m}}=176.2^{\circ} \mathrm{C}$. The average molecular weight of the PCL pellets is $M_{\mathrm{w}}=1.4 \times 10^{5} \mathrm{~g} / \mathrm{mol}$, the glass transition temperature $T_{\mathrm{g}}=-60^{\circ} \mathrm{C}$ and the melting point $T_{\mathrm{m}}=60^{\circ} \mathrm{C}$. The mixing ratio of PLA and PCL was 90/10, 80/20, 70/30. PLA was press-processed using a conventional hot press at $190^{\circ} \mathrm{C}$ and $30 \mathrm{MPa}$ for $30 \mathrm{~min}$. After melt-mixing in a conventional melt-mixer at $190^{\circ} \mathrm{C}$ for $20 \mathrm{~min}$ and at a rotor speed of $50 \mathrm{rpm}$, the blend mixtures were press-processed using a conventional hot press at $190^{\circ} \mathrm{C}$ and $30 \mathrm{MPa}$ for $30 \mathrm{~min}$. Photographs of specimen surfaces after polishing and cryo-fractured surfaces are shown in Figure 1. PCL particle size becomes larger with increasing PCL content. DSC thermograms of PLA and PLA/PCL blends are shown in Figure 2. Peaks near $170^{\circ} \mathrm{C}$ showing the melting point of PLA were observed. The peaks near $90^{\circ} \mathrm{C}$ show the crystallization of PLA.

Compressive test specimens were made using a turning machine, and their end faces were polished and parallelized. We used dynamic compressive test specimens with a diameter of approximately $13 \mathrm{~mm}$, so that stress-strain curves could be accurately measured using our equipment. Specimen thickness was $4 \mathrm{~mm}$. In the quasi-static tests, based on ASTM D695-02a, we mainly used specimens with a diameter of $6 \mathrm{~mm}$ and a thickness of $9 \mathrm{~mm}$. In order to examine the effect of specimen size on stress-strain curves, we also used specimens with a diameter of $5 \mathrm{~mm}$ and a thickness of $5 \mathrm{~mm}$.

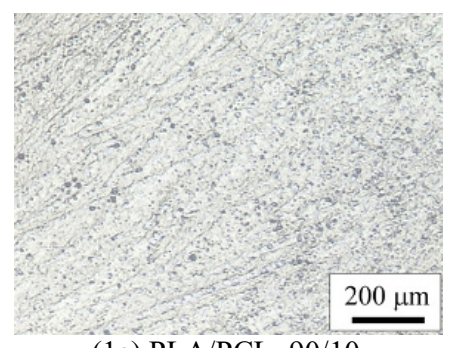

(1a) $\mathrm{PLA} / \mathrm{PCL}=90 / 10$

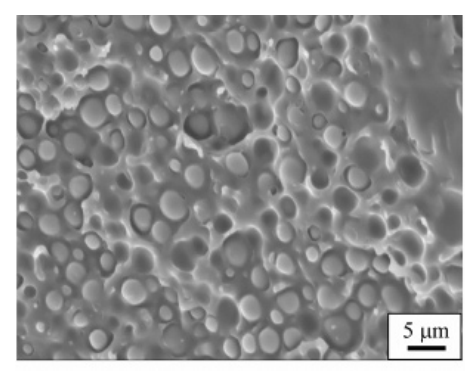

(2a) PLA/PCL $=90 / 10$

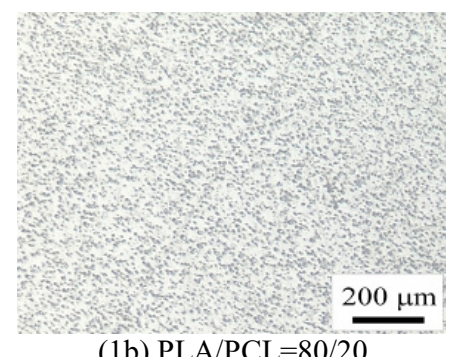

(1b) $\mathrm{PLA} / \mathrm{PCL}=80 / 20$

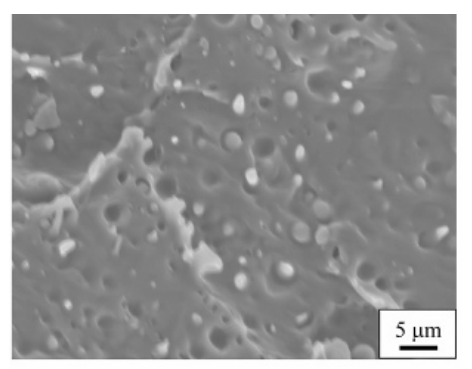

(2b) $\mathrm{PLA} / \mathrm{PCL}=80 / 20$

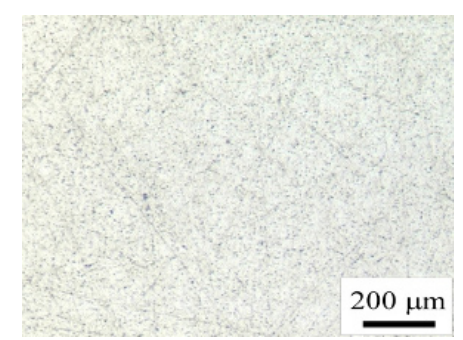

(1c) $\mathrm{PLA} / \mathrm{PCL}=70 / 30$

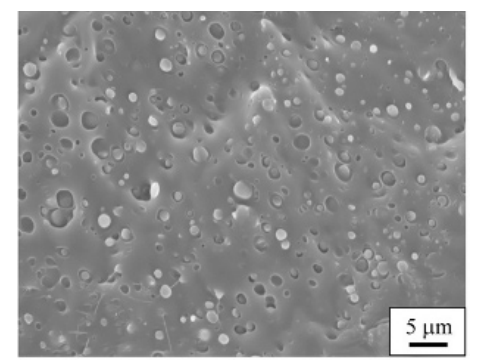

(2c) $\mathrm{PLA} / \mathrm{PCL}=70 / 30$

Figure 1. Photographs of specimen surfaces after polishing $(1 \mathrm{a}-\mathrm{c})$ and cryo-fractured surfaces $(2 \mathrm{a}-\mathrm{c})$.

\subsection{Compressive tests}

In the quasi-static compressive tests of strain rates from $10^{-4}$ to $10^{-1} \mathrm{~s}^{-1}$, we mainly used a universal testing machine (Mori Kikai Seisakusho Co.). At high strain rates of $10^{2}$ to $10^{3} \mathrm{~s}^{-1}$, compressive properties were examined by the split Hopkinson pressure bar (Kolsky bar) shown in Figure 3. The input and output bars were made of aluminum alloy A2024-T4, and their diameter and length were $28 \mathrm{~mm}$ and $1900 \mathrm{~mm} / 1300 \mathrm{~mm}$, respectively. Strain gages were applied to both sides of the input bar $950 \mathrm{~mm}$ away from the specimen and the output bar $300 \mathrm{~mm}$ away from the 


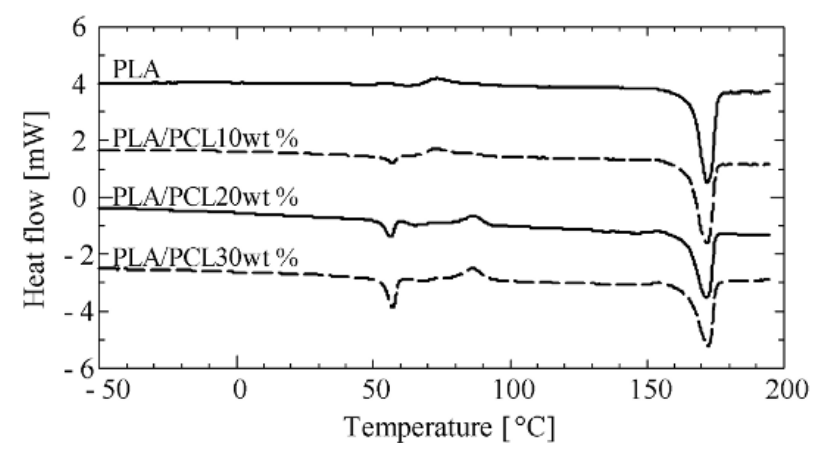

Figure 2. DSC thermograms of PLA and PLA/PCL blends.

specimen. We used silicone grease lubricant between the bars and specimens to reduce the frictional restraint. Because the stress histories were almost the same on both ends of specimens, the strain and stress of specimens were calculated from the strain of bars measured by the strain gages based on the following equations [6]:

$$
\begin{gathered}
\varepsilon(t)=\frac{2 c_{3}}{L} \int_{0}^{t}\left[\varepsilon_{I}(t)-\varepsilon_{T}(t)\right] d t \\
\sigma(t)=\frac{A E}{A_{S}} \varepsilon_{T}(t)
\end{gathered}
$$

where $\varepsilon_{I}$ and $\varepsilon_{T}$ are the axial strain in the input bar induced by the incident wave and the axial strain in the output bar induced by the transmitted wave. $E$ and $c_{3}$ are Young's modulus and elastic wave velocity of the input and output bars, respectively. $L$ is the specimen thickness. $A$ and $A_{S}$ are the areas of cross sections of the input/output bars and the specimens. Material constants of the aluminum alloy (A2024-T4) bars we used in the calculation are shown in Table 1. We used brass strikers with a diameter of $20 \mathrm{~mm}$ and length of 220 to $390 \mathrm{~mm}$. During the experiments, the humidity in our laboratory was 15 to $65 \%$. Specimens were maintained at a temperature of 23 to $25^{\circ} \mathrm{C}$ using silicone rubber heaters.

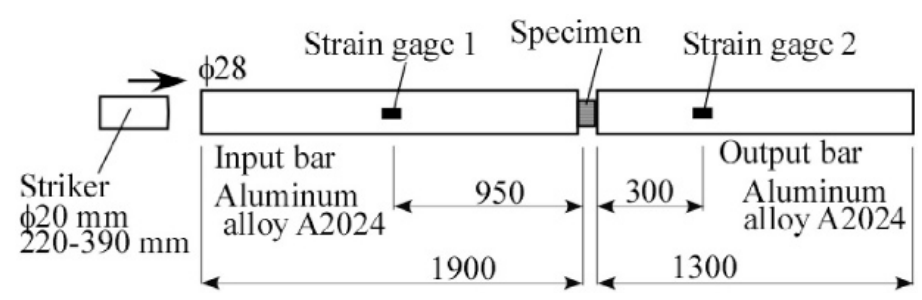

Figure 3. Experimental setup for split Hopkinson pressure bar method.

Table 1. Material constants of input and output bars used in calculation.

\begin{tabular}{|l|c|c|}
\hline Density & $\begin{array}{c}\text { Elastic wave velocity } \\
\text { in the bar, } c_{3}\end{array}$ & Young's modulus $E$ \\
\hline $2.77 \times 10^{3} \mathrm{~kg} / \mathrm{m}^{3}$ & $5150 \mathrm{~m} / \mathrm{s}$ & $73.6 \mathrm{GPa}$ \\
\hline
\end{tabular}




\section{RESULTS AND DISCUSSION}

At first, the result of a quasi-static compressive test for $\mathrm{PLA} / \mathrm{PCL}=80 / 20$ specimen is shown in Figure 4. The cross head speed of the universal testing machine was $0.6 \mathrm{~mm} / \mathrm{min}$ (strain rate $1.0 \times 10^{-3} \mathrm{~s}^{-1}$, temperature $23^{\circ} \mathrm{C}$, humidity $15 \%$ ). First, the stress increased with strain. Second, the nominal stress-strain curve showed a peak in the vicinity of the elastic limit. Third, the stress decreased slightly with strain. The stress remained steady at $53 \mathrm{MPa}$. Young's modulus at a strain rate of $1.0 \times 10^{-2} \mathrm{~s}^{-1}$ was determined to be $2.5 \mathrm{GPa}$ by the collinear approximation of the stressstrain curve below a strain of 0.015 (nominal stress approximately $32 \mathrm{MPa}$ ).

Next, the dynamic properties of PLA/PCL $=80 / 20$ specimen were examined using the split Hopkinson pressure bar method. Figure 5 (a) shows the strain history and stress history of the specimen obtained from the strain gages using Equation (1) and (2). It was found from the strain history that the strain increased at an increasing rate from 10 to $210 \mu \mathrm{s}$ after the onset of the dynamic loading. The stress history increased rapidly up to $80 \mu$ s after the onset of the dynamic loading. After that, even though the strain increased at an increasing rate, the stress decreased slightly. The stress-strain curve and strain rate-strain curve obtained from Figure 5 (a) are plotted in Figure 5 (b). The stress-strain curve at high strain rates showed a peak near the elastic limit and, after that, because of polymer relaxation the stress decreased gradually with increasing stress. Because the strain rate slightly changed during compression, the strain rate was determined by the averaged value of the strain rate-strain curve [7]. In Figure 5, the strain rate was $720 \mathrm{~s}^{-1}$.

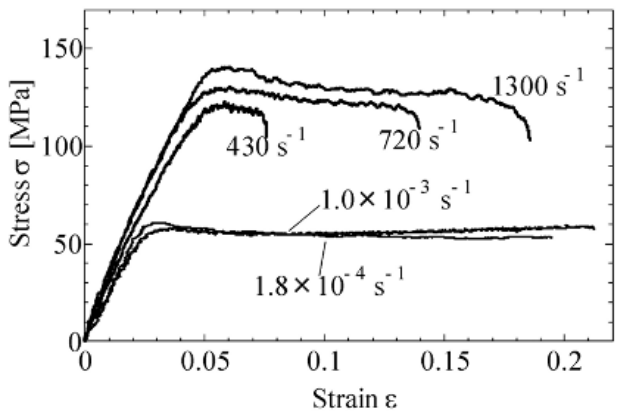

Figure 4. Comparison of stress-strain curves with respect to strain rate (PLA/PCL $=80 / 20)$.

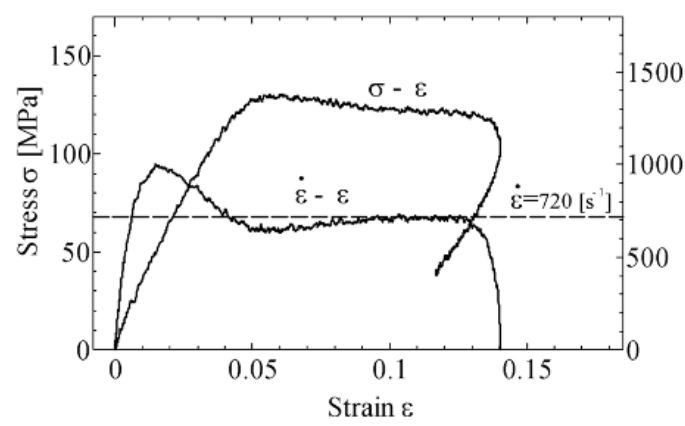

(a) Stress and strain histories

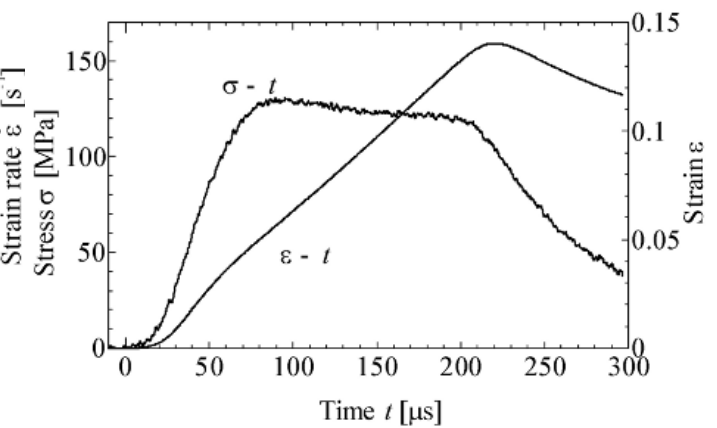

(b) Stress-strain curve and strain rate-strain curve

Figure 5. Stress history and strain history of PLA/PCL $=80 / 20$ at a strain rate of $720 \mathrm{~s}^{-1}$ (Temperature $20^{\circ} \mathrm{C}$, Humidity $39 \%$ ). 


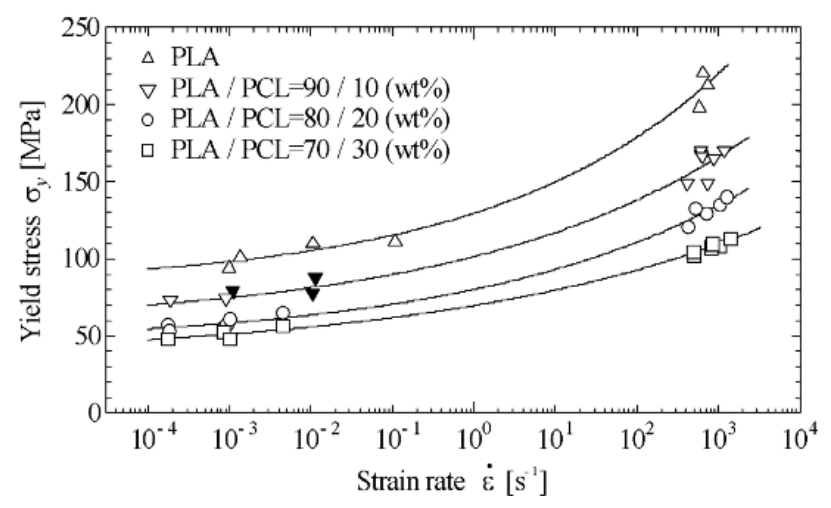

Figure 6. Relationship between yield stress and strain rate (Black triangle in figure shows specimens with a diameter of $5 \mathrm{~mm}$ and a thickness of $5 \mathrm{~mm}$ ).

Including the dynamic stress-strain curve shown in Figure 5 (b), the stress-strain curves at strain rates of $1.8 \times 10^{-4} \mathrm{~s}^{-1}, 430 \mathrm{~s}^{-1}$ and $1300 \mathrm{~s}^{-1}$ were added to Figure 3 . The flow stress and Young's modulus increased with the strain rate as is commonly seen in engineering plastics. In most of the strain rate, because the stress-strain curve showed a peak near the elastic limit, we compared the yield stresses (maximum flow stresses) of the stress-strain curve, as shown in Figure 6. In the present study, yield stress of all experimental results increased linearly with the strain rate up to a strain rate of $10^{0} \mathrm{~s}^{-1}$. At the strain rate of approximately $10^{0} \mathrm{~s}^{-1}$, the increasing rate of yield stress changed because of the change in polymer relaxation processes as can be seen in polymethyl methacrylate (PMMA) and polycarbonate (PC) [8].

Figure 7 shows the effect of PCL content on stress-strain curves at high strain rates of 720$870 \mathrm{~s}^{-1}$. The yield stress and Young's modulus decreased with increasing PCL content. The strain showing yield stress (maximum stress) for each curve was almost the same. The effect of PCL content on the yield stress is shown in Figure 8. Regardless of strain rate, the yield stress decreased with increasing PCL content. However, the reduction of yield stress was not proportional to the PCL content. The reduction rate of yield stress decreased with increasing PCL content. When the PCL content is $30 \%$, the yield stress was half of that for PLA specimens. The main reason for this is that the size of PCL was not the same for specimens of PLA/PCL $=90 / 10,80 / 20$ and 70/30 as shown in Figure $1(2 \mathrm{a}-\mathrm{c})$. The peak near the elastic limit became unclear with increasing PCL content. In the case of PLA/PCL $=70 / 30$, we could barely see a small peak.

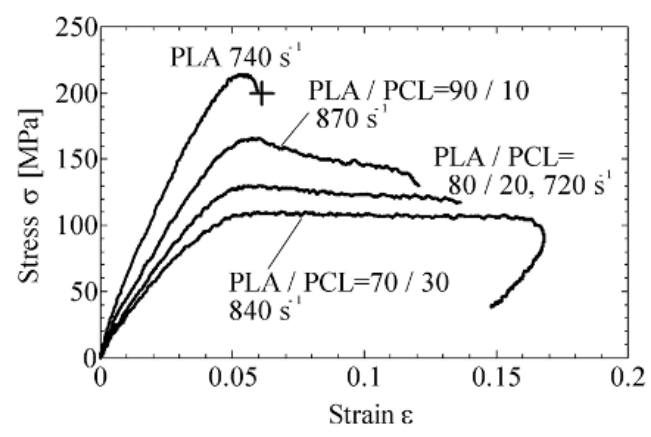

Figure 7. Effect of PCL content on stress-strain curves at high strain rates $\left(720-870 \mathrm{~s}^{-1}\right)$. 


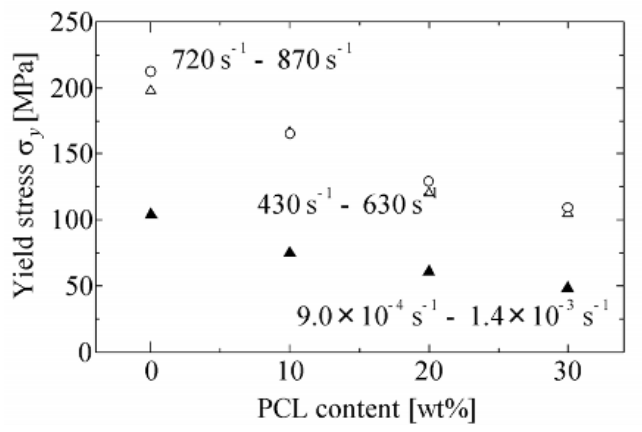

Figure 8. Effect of PCL content on the yield stress.

Finally, we thought that the trend of yield stress shown in Figure 6 could be described by the Cowper-Symonds equation [9, 10]:

$$
\frac{\sigma_{d}}{\sigma_{s}}=1+\left(\frac{\dot{\varepsilon}}{D}\right)^{\frac{1}{p}}
$$

Here, $\sigma_{d}$ and $\sigma_{s}$ are the dynamic yield stress at a strain rate $\dot{\varepsilon}$ and the associated static yield stress. $D$ and $p$ are constants for a particular material. By fitting Equation (3) to the experimental results shown in Figure $6, \sigma_{s}, D$ and $p$ were derived. These values for four types of PLA are shown in Table 2. The value of $D$ decreased with increasing PCL content. This means that the strain rate dependency of yield stress was small when PLA/PCL $=70 / 30$.

Table 2. Constants of Equation (3) for PLA and PLA/PCL blends.

\begin{tabular}{|l|c|c|c|}
\hline & $\sigma_{s}[\mathrm{MPa}]$ & $D\left[\mathrm{~s}^{-1}\right]$ & $p$ \\
\hline PLA & 82 & 33 & 6.5 \\
\hline PLA/PCL $=90 / 10$ & 56 & 5.2 & 7.8 \\
\hline PLA/PCL $=80 / 20$ & 44 & 4.5 & 7.5 \\
\hline PLA/PCL $=70 / 30$ & 35 & 1.2 & 9.0 \\
\hline
\end{tabular}

\section{CONCLUSIONS}

We examined the effect of PCL content on the stress-strain curves using the split Hopkinson pressure bar system and a universal testing machine. The yield stress and Young's modulus decreased with increasing PCL content. However, the reduction of yield stress was not proportional to PCL content. The values of constants in the Cowper-Symonds equation with respect to yield stress were determined for PLA and PLA/PCL polymer blends.

\section{Acknowledgments}

This work was supported in part by the Collaborative Research Program of the Research Institute for Applied Mechanics, Kyushu University. We thank Mr. Noriomi Ito, a master course student of Nagoya Institute of Technology, for generously assisting with the experiments.

\section{References}

[1] Tsuji H. and Ikeda Y., J. Appl. Polym. Sci., 70 (1996), pp. 2367-2375.

[2] Chen C.C., Chueh J.Y., Tseng H., Huang H.M. and Lee S.Y., Biomaterials, 24 (2003), pp. 1167-1173. 
[3] Todo M., Park S.-D., Takayama T. and Arakawa K., Eng. Fract, Mech., 74 (2007), pp. 1872-1883.

[4] Todo M. and Takayama T., Interface Oral Health Science, (2007), pp. 95-104.

[5] Hiljanen-Vainio M., Varpomaa P., Seppälä J. and Törmälä P., Macromo. Chem. Phys., 197 (1996), pp. 1503 - 1523.

[6] Gray G.T. III, ASM Int., 8 (2000), pp. 462-476.

[7] Nakai K. and Yokoyama T., J. Solid Mech. Mater. Eng. 2 (2008), pp. 557-566.

[8] Bauwens-Crowet C., Bauwens J.C., and Homes G., J. Polym. Sci., 2 (1969), pp. 735-742.

[9] Jones N., Structural Impact (Cambridge University Press, 1989) pp. 348-352.

[10] Børvik T., Hanssen A.G., Dey S., Langberg H. and Langseth M., Eng. Struct., 30 (2008), pp. 1605-1620. 